\title{
Plasmonic Antennas Nanocoupler for Telecom Range: Simulation, Fabrication and Near-Field Characterization
}

Andryieuski, Andrei; Malureanu, Radu; Lavrinenko, Andrei; Zenin, Vladimir A.; Volkov, Valentyn S.; Bozhevolnyi, Sergey I.

\section{Published in:}

Proceedings of 2014 Conference on Lasers and Electro-Optics (CLEO)

Publication date:

2014

Document Version

Publisher's PDF, also known as Version of record

Link back to DTU Orbit

Citation (APA):

Andryieuski, A., Malureanu, R., Lavrinenko, A., Zenin, V. A., Volkov, V. S., \& Bozhevolnyi, S. I. (2014).

Plasmonic Antennas Nanocoupler for Telecom Range: Simulation, Fabrication and Near-Field Characterization.

In Proceedings of 2014 Conference on Lasers and Electro-Optics (CLEO) IEEE.

\section{General rights}

Copyright and moral rights for the publications made accessible in the public portal are retained by the authors and/or other copyright owners and it is a condition of accessing publications that users recognise and abide by the legal requirements associated with these rights.

- Users may download and print one copy of any publication from the public portal for the purpose of private study or research.

- You may not further distribute the material or use it for any profit-making activity or commercial gain

- You may freely distribute the URL identifying the publication in the public portal 


\title{
Plasmonic Antennas Nanocoupler for Telecom Range: Simulation, Fabrication and Near-Field Characterization
}

\author{
Andrei Andryieuski, Radu Malureanu and Andrei V. Lavrinenko \\ DTU Fotonik, Technical University of Denmark, Oersteds pl. 343, Kongens Lyngby DK-2800, Denmark \\ andra@fotonik.dtu.dk \\ Vladimir A. Zenin, Valentyn S. Volkov and Sergey I. Bozhevolnyi \\ Institute of Technology and Innovation, University of Southern Denmark, Niels Bohrs Alle 1, Odense M DK-5230, Denmark
}

\begin{abstract}
We report simulation, fabrication and, for the first time, full amplitude-phase near-field optical characterization in telecom range of the compact and efficient plasmonic nanoantenna based couplers. Near-field data allowed characterizing the subwavelength slot waveguide's propagation losses and effective mode index that correspond well to the simulated ones.

OCIS codes: (180.4243) Near-field microscopy; (250.5403) Plasmonics; (240.6680) Surface plasmons.
\end{abstract}

Plasmonic waveguides allow for subwavelength mode localization, but with an advantage of tight waveguide integration, the challenge of light coupling into the plasmonic waveguide appears. Various solutions can be applied [1] including gratings, tapered waveguide sections, directional coupling and nanoantennas. Among these approaches plasmonic antennas are the most compact. Nanoantenna as coupler to plasmonic slot waveguide was suggested theoretically [2-5] and then measured experimentally with cross-polarization microscopy [6-7] in telecom and with near-field microscopy in visible [8] and mid-infrared [9] ranges. Nevertheless, near-field characterization of the plasmonic antenna nanocouplers for telecom range has not been demonstrated so far. In this contribution we present, for the first time, amplitude and phase measurements in the telecom range of the propagating plasmons in the slot waveguide excited with a plasmonic antenna [see Fig. 1(a)].

(a)

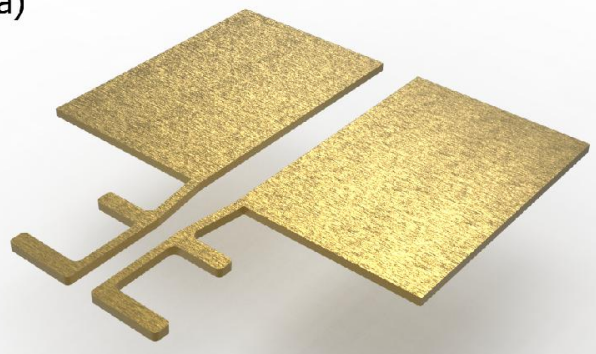

(b)

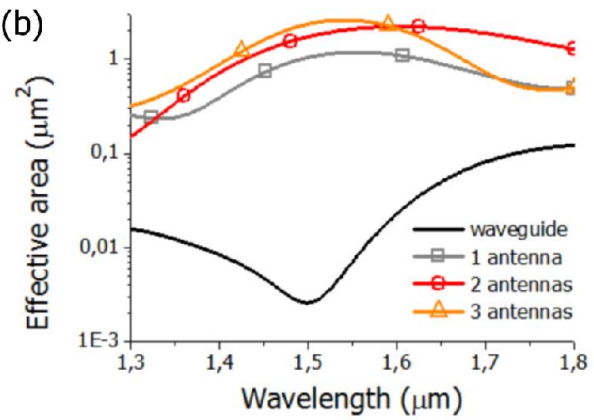

Fig. 1. (a) Plasmonic nanocoupler, consisting of two serial dipole antennas, connected to plasmonic slot waveguide. (b) Effective area of bare waveguide termination (black line), one (grey squares), two (red circles) and three (orange triangles) serially connected antennas.

Antenna coupler demonstrates tremendous coupling efficiency increase of 1000 times compared to the waveguide termination.

Simulation and optimization of the antenna nanocoupler was done in CST Microwave Studio. Effective area (figure-of-merit for the nanocoupler equal to the ratio of coupled power to an incident plane wave's power flux) of a bare waveguide termination, one, two and three serially connected antennas are shown in the Fig. 1(b). At the wavelength $1.5 \mu \mathrm{m}$ three serially connected antennae demonstrate nearly 1000 times better effective area and thus coupling efficiency compared to the case without antenna coupler.

(a)

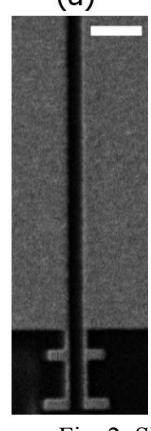

(b)

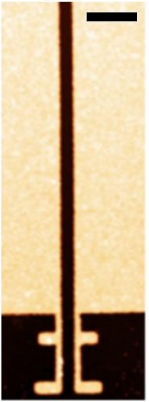

(d)

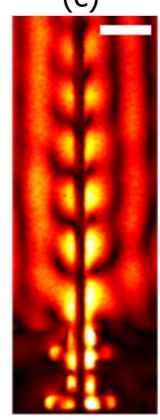

(e)

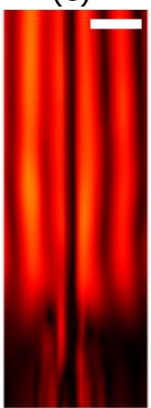

(f)

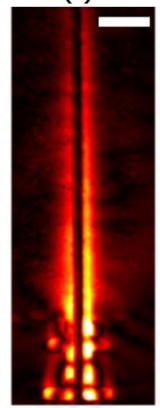

(g)

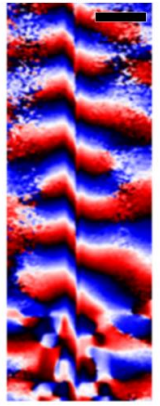

(h)

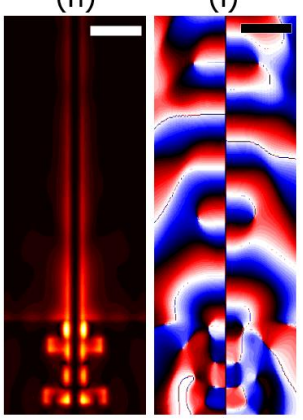
(d) phase; (e) amplitude of the background SPPs; (f) amplitude and (e) phase of the slot mode after filtration. The latter correspond well to the numerically modeled amplitude (h) and phase (i). Scale bars are equal to $1 \mu \mathrm{m}$. 
Fabrication of the sample on double polished $500 \mu \mathrm{m}$ thick borofloat glass substrate was done with e-beam lithography (ZEP520 resist spinning, $10 \mathrm{~nm} \mathrm{Al} \mathrm{thermal} \mathrm{evaporation,} \mathrm{e-beam} \mathrm{exposure} \mathrm{in} \mathrm{JEOL} \mathrm{JBX-9300FS} \mathrm{and}$ development), gold evaporation (Wordentec QCL 800) and lift-off. The sample was then characterized with scanning electron microscope [Fig. 2 (a)] and atomic force microscope [Fig. 2 (b)]. The plasmon excitation was done with the $\mathrm{CW}$ tunable laser and measurements were performed with the scattering-type scanning near-field optical microscope in transmission mode (s-SNOM, Neaspec). Measured amplitude [Fig. 2 (c)] and phase [Fig. 2 (d)] of the raw field (component perpendicular to the sample's surface) is formed by the interference pattern between the propagating slot plasmon and surface plasmons excited upon wave's diffraction through the slot. In order to extract the slot plasmon field we filtered out the surface plasmon field [Fig. 2(e)] that is relatively constant along the slot. The filtered experimental field [Fig. 2 (f-g)] is in a good correspondence with the simulation results [Fig. 2 (h-i)].
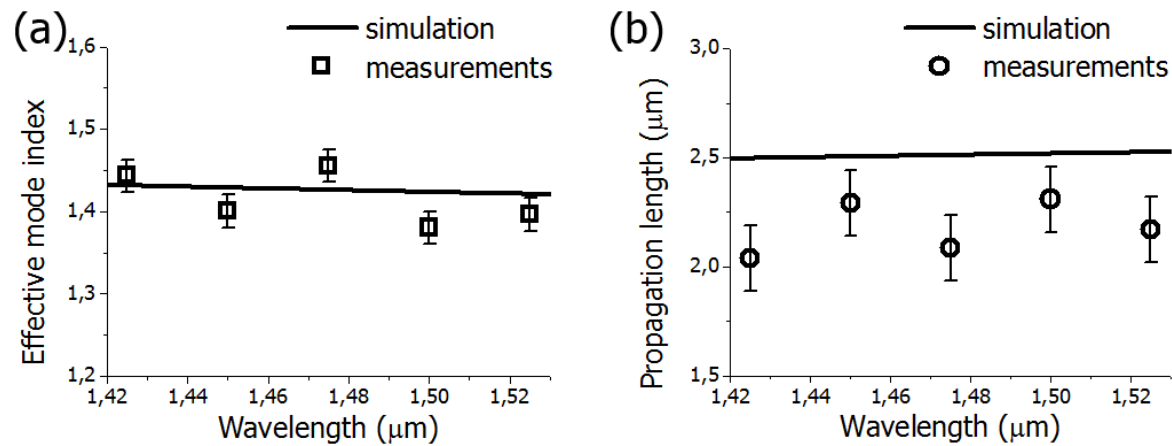

Fig. 3. Simulated (solid lines) and measured (symbols) effective mode index (a) and propagation losses (b) of the plasmonic slot waveguide.

From the measurements of amplitude decay [Fig. 2 (f)] and phase advance [Fig. 2 (g)] at various wavelengths we were able to characterize the effective mode index [Fig. 3 (a)] and propagation length [Fig. 3 (b)] of the plasmonic slot waveguide. The measured effective index corresponds well to the simulated one, while measured propagation length is smaller than the simulated one that can be attributed to the higher losses due to fabricated sample imperfections. We have also confirmed experimentally that the plasmonic antennas are an efficient and compact device for telecom range in-coupling into the subwavelength plasmonic slot waveguide.

\section{References}

[1] A. Andryieuski and A. V. Lavrinenko, "Nanocouplers for Infrared and Visible Light,” Adv. Optoelectron. 2012, 1-17 (2012).

[2] J. Wen, S. Romanov, and U. Peschel, “Excitation of plasmonic gap waveguides by nanoantennas,” Opt. Express 17, 5925-5932 (2009).

[3] J. Huang, T. Feichtner, P. Biagioni, and B. Hecht, "Impedance matching and emission properties of nanoantennas in an optical nanocircuit," Nano Lett. 9, 1897-1902 (2009).

[4] Z. Fang, Y. Lu, L. Fan, and C. Lin, "Surface Plasmon Polariton Enhancement in Silver Nanowire - Nanoantenna Structure,” Plasmonics 5 $57-62(2010)$

[5] A. Andryieuski, R. Malureanu, G. Biagi, T. Holmgaard, and A. Lavrinenko, "Compact dipole nanoantenna coupler to plasmonic slot waveguide.," Opt. Lett. 37, 1124-6 (2012).

[6] J. Wen, P. Banzer, A. Kriesch, D. Ploss, B. Schmauss, and U. Peschel, "Experimental cross-polarization detection of coupling far-field light to highly confined plasmonic gap modes via nanoantennas,” Appl. Phys. Lett. 98, 101109 (2011).

[7] A. Kriesch, S. P. Burgos, D. Ploss, H. Pfeifer, H. A Atwater, and U. Peschel, "Functional plasmonic nanocircuits with low insertion and propagation losses.," Nano Lett. 13, 4539-45 (2013).

[8] Z. Fang, L. Fan, C. Lin, D. Zhang, A. J. A. J. Meixner, and X. Zhu, "Plasmonic Coupling of Bow Tie Antennas with Ag Nanowire," Nano Lett. 11, 1676-1680 (2011).

[9] M. Schnell and P. Alonso-Gonzalez, "Nanofocusing of mid-infrared energy with tapered transmission lines,” Nat. Photon. 5, 283-287 (2011). 